\title{
LA MERCANTILIZACIÓN DE LA EDUCACIÓN COMO ESCENARIO MUNDIAL DEL ESPACIO EUROPEO DE EDUCACIÓN SUPERIOR
}

\author{
(UNIVERSITIES AS MARKETPLACES IN THE EUROPEAN HIGHER \\ EDUCATION AREA)
}

Florentino Sanz Fernández

UNED

\section{RESUMEN}

En la actualidad, las políticas sociales y de servicios públicos están sometidas a fuertes presiones. La universidad, por su parte, se orienta cada vez más hacia el modelo de mercado. Todos los países están sometidos a grandes presiones para poder satisfacer la demanda de conocimiento superior que necesitan los sistemas productivos. Los centros de formación superior son cada día más conscientes de que se están dirigiendo a consumidores que pueden elegir los mejores cursos existentes en el mercado. En Europa, el Espacio Europeo de Educación Superior también se orienta en este sentido y esto se ve en la documentación que aparece sobre enseñanza superior. Sin embargo, las universidades deben enseñar también a pensar y por qué no a imaginar, a sentir y a soñar, porque aunque estas capacidades no se necesiten para producir, se necesitan para vivir con dignidad.

\section{ABSTRACT}

Current social policies and public services are under great pressure. On the other hand, universities get increasingly along with the free market. All countries are under strong pressure to satisfy the demand for highly specialised knowledge that productive systems require. Universities are increasingly aware that they are addressing consumers who are able to choose amongst the world's best courses. The European Space for Higher Education is also walking this line, which can be traced in several documents on higher education. However, universities must also train their students in some abilities other than market oriented ones: critical thinking, creative thinking and emotional intelligence. These may not be useful in a productive job, but they are necessary to live with dignity. Finally, we reflected on the resurgence of the economic approaches to education, and the conclusion at which we arrived, point towards of the necessity of an interdisciplinary aproach to answer the questions that historically the economy of education has been formulating. 


\section{INTRODUCCION}

En Europa se están movilizando todas las instituciones para generar una profunda transformación de los estudios superiores en toda la región. El objetivo de esta movilización, como muy bien defiende el profesor José Luis Pardo (2005), no es ni científico, ni político sino económico. "Hasta en público reconocen sus promotores y defensores que este proyecto se apoya en una razón exclusivamente económica: la necesidad de competir con los Estados Unidos también en el mercado de la educación".

La Universidad española se encuentra en este momento ante el desafío de afrontar una reforma exigida sólo por la necesidad de adaptarse al denominado Espacio Europeo de Educación Superior (EEES). En este caso no se trata de una reforma científica puesto que no se han detectado deficiencias ni obsolescencia en la docencia o en la investigación. Tampoco se trata de una necesidad política. El objetivo fundamental parece ser el declarado en la estrategia de Lisboa, el de preparar profesionales y adaptar las institución universitaria para competir con los Estados Unidos en el mercado de la educación y en convertir a la región europea en la región más competitiva del planeta. Se trata por lo tanto de conseguir, con esta reforma, un objetivo de tipo económico. Por todo ello, nos parece muy conveniente ofrecer algunas reflexiones sobre el contexto mundial en el que este cambio se produce.

Pero antes de adentrarnos en el ámbito específico de la educación hagamos una rápida exposición sobre la evolución que está ocurriendo en el ámbito de las políticas sociales y de los servicios públicos.

\section{DEL MODELO DE ESTADO SOCIAL HACIA UN MODELO DE ESTADO NEOLIBERAL}

El Consenso de Washington, sintagma acuñado por John Williamson para sintetizar las propuestas de política económica que dominaban en la capital norteamericana a fines de los años 80 , se ha convertido en un referente histórico que ha incidido en la evolución de las políticas sociales ya que supone el comienzo del abandono de las denominadas políticas de los Estados Sociales y la apuesta por una fuerte ofensiva ideológica a favor del libre mercado. En realidad el Consenso de Washington potencia políticas que tienden a delegar en el Mercado muchas de las tareas que venía afrontando directamente el Estado en el modelo del Estado Social. A partir del Consenso de Washington se considera que la privatización de empresas de propiedad estatal no sólo constituye una fuente de ingresos a corto plazo para el Estado sino que, a largo plazo, se le libera de la responsabilidad de financiar ulteriores inversiones. La lógica de las privatizaciones obedece a la creencia de que la industria privada se administra más eficientemente que la empresa estatal. El nuevo modelo de la gestión pública no propugna quizás, como suele decirse, una política de desaparición del Estado, pero sí una política de los Estados orientada hacia el máximo del Mercado y hacia el mínimo de Estado. 
En los países que van introduciendo las políticas de Mercado, la diferencia entre gasto público y el gasto privado en los servicios sociales va aumentando significativamente, como podemos observar en el ejemplo siguiente.

GASTO PÚBLICO Y PRIVADO EN PROTECCIÓN SOCIAL, 1990 $\%$ del PIB

Suecia

EE.UU

Gasto público social

Gastos tributarios

$33.1 \quad 14.6$

Gastos privados educación

0.0

Gastos privados salud

0.1

2.5

Gastos privados pensiones

1.1

2.5

Gasto total en protección social

35.5

\% de Gastos del Hogar

Gastos privados en Educación, Salud y Pensiones

Fuente: G. ESPING - ANDERSEN (2000) citado por Alejandro Foxley en ¿Más mercado o más estado para América latina?, artículo preparado para el Seminario del BID "Nuevas Fronteras de la Reforma del Estado", Lima, Perú, Marzo 2004.

http://www.iadb.org/sds/doc/SGC-FOXLEY.pdf

Entre Suecia (modelo de Estado Social) y EEUU. (Modelo de Mercado) la diferencia del gasto total en protección social representa seis puntos (de 29'6 a $35^{\prime} 5$ ). Una diferencia que, siendo significativa, se queda corta si comparamos el origen de los gastos: el 33 '1 \% es gasto público en el modelo de Estado mientras, en el modelo de Mercado, se reduce al 14'6\%. Por otra parte los ciudadanos del Estado Social pagan 16 '8 puntos más en concepto de impuestos que los del Estado Liberal. Eso quiere decir que muchos de los servicios en protección social han de ser comprados a título individual por los particulares en el modelo del Mercado, mientras que en el Modelo de Estado social los servicios son ofrecidos por igual a todos y pagados con una mayor cantidad de impuestos. De hecho, los gastos privados en educación, salud y pensiones son 16'1 puntos más en la sociedad americana que en la sueca.

Este proceso mercantilizador, aunque más lentamente que en Estados Unidos, también tiene su arraigo en Europa. Si queremos reflexionar sobre la evolución del estado social en Europa, y concretamente en España, nos veríamos obligados a consultar los estudios de Vicente Navarro (2006), uno de los analistas que mejor ha estudiado el estado social en España. Aportamos solamente algunos datos que dicho autor nos ofrece. 


\begin{tabular}{|l|l|l|}
\hline \multicolumn{3}{|c|}{ Ingresos al Estado (como porcentaje del PIB) } \\
\hline & 1993 & 1999 \\
\hline España & $38,1 \%$ & $39,9 \%$ \\
\hline UE-15 & $44,1 \%$ & $46,4 \%$ \\
\hline \multicolumn{3}{|c|}{ Gasto en protección social (\% del PIB) } \\
\hline & 1993 & 2000 \\
\hline España & $24,0 \%$ & $20,1 \%$ \\
\hline UE-15 & $28,8 \%$ & $27,3 \%$ \\
\hline
\end{tabular}

Fuente V. Navarro (2002)

Si tenemos en cuenta que el año 1975, cuando murió Franco, el porcentaje del PIB en gasto social era solamente del 14\%, cuando en Europa ya se empleaba el $22 \%$, el avance en positivo que se ha dado en España durante el proceso democrático ha sido significativo. No obstante hay que observar que precisamente en el momento en el que estaba creciendo el Estado de Bienestar en España, y cuando todavía distábamos mucho de igualarnos con el resto de Europa, se produce un frenazo en los inicios de los años noventa como podemos observar en las tablas anteriores. El porcentaje del PIB empleado en protección social ha ido disminuyendo desde 1993 al año 2000 tanto en España como en Europa, pasando de ser, en España,

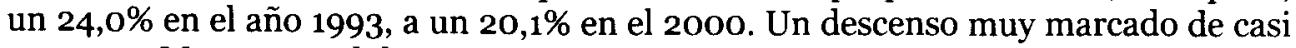
4 puntos. Menos que el descenso europeo de sólo 1,5 puntos, de 28,8 a 27,3.

Si comparamos España con Suecia (uno de los países más convencidos, hasta ahora, del modelo del estado social) la diferencia nos parece notable en algunos de los indicadores más significativos:

- En España, el 5,9\% de la población adulta trabaja en sanidad, educación y servicios de ayuda a la familia (año 200o, último año para el cual existen datos comparables para los países de la UE). El promedio de la UE-15,11\%. En Suecia, tal porcentaje alcanza un $16,9 \%$

- El porcentaje del PIB empleado en servicio sociales (año 2000) es un 20,1\% el promedio de la UE-15, es de un $27,3 \%$. En Suecia, tal porcentaje es 32,3

- El gasto público educativo español como porcentaje del PIB es 4,3\%. El promedio de la UE-15, 5,4\% del PIB.

Según Vicente Navarro (2004), una explicación, un tanto complaciente, a esta diferencia es que el PIB español ha crecido más rápidamente que el del promedio de la UE y que, por lo tanto, aun cuando el porcentaje de tal gasto público social sobre el PIB en España ha ido descendiendo, el gasto absoluto, sin embargo, ha crecido 
durante los años noventa. En apoyo de esta tesis se señala que el gasto público social en España pasó de 100.849 millones de euros en 1993 a 122.525 millones en 2000 , incrementándose por lo tanto en 21.675 millones. La diferencia no obstante entre España y el resto de Europa en gasto social sigue siendo notable como podemos ver en la tabla siguiente. Quien, en definitiva, asume este déficit de gasto público en bienes sociales es el mercado y las familias que tienen que pagar sus productos.

Gasto en protección social por habitante (en unidades de poder de compra)

Fuente: V. Navarro (2004 y 2006).

\begin{tabular}{|l|l|l|l|}
\hline & 1993 & 2000 & 2002 \\
\hline ESPAÑA & 3.049 & 3.713 & 4.089 \\
\hline UE-15 & 4.668 & 6.155 & 6.472 \\
\hline
\end{tabular}

\section{LA EVOLUCIÓN DE LA EDUCACIÓN SUPERIOR EN EL ESCENA- RIO DE LAS POLITICAS DE MERCADO}

Si realizáramos un rápido recorrido a través de la historia de la educación podríamos tipificar al menos cuatro grandes mutaciones que han alterado los modelos de enseñar durante los últimos siglos. En este momento nos encontraríamos en la cuarta mutación, caracterizada, según varios analistas entre los que cabe destacar Brunner (2005)', Niko Hirt (2003) y Laval (2004), por la mercantilización y la industrialización de la enseñanza.

- La primera mutación está marcada por el establecimiento de la enseñanza como función social específica. Es una mutación que transita desde el modelo educativo familiar y comunitario, un tanto informal y desorganizado, a otro modelo más metódico y del que se ocupan determinadas instituciones como la escuela y determinados profesionales específicamente (aunque no siempre exclusivamente) dedicados a la enseñanza.

- La segunda mutación se caracteriza por la politización de la enseñanza. Se produce en un momento en el que todo un conjunto de instituciones, explícita y exclusivamente dedicadas a la enseñanza se articulan para desempeñar una función conjunta en un Estado o en una nación. Es la época en la que nacen los sistemas nacionales de enseñanza, abandonando los paradigmas educativos privados, particulares y religiosos para centrarse en un paradigma público, secularizado y homogéneo. Su objetivo prioritario es la construcción social del ciudadano.

- La tercera mutación está marcada por la masificación de los sistemas de enseñanza que demanda el nuevo contexto de la revolución industrial, caracterizada por aglutinar grandes masas de trabajadores en torno a las fá- 
bricas y por el tránsito de la población de los contextos rurales a los urbanos. Estas dos nuevas situaciones van a requerir aprendizajes masivos, sobre todo de disciplina laboral que enseñe a los obreros a trabajar de forma muy diferente a como se hacía en los gremios y de disciplina urbana que enseñe a los rurales a convivir en los masificados medios urbanos. William Harris expresó con toda claridad que en la sociedad industrial moderna, la conformidad con el tiempo del ferrocarril, con el comienzo del día laboral en la fábrica y con otras características de la vida en la ciudad requerían total precisión y regularidad que debían aprenderse en la escuela, exigiendo al alumno cumplir con sus deberes en el tiempo fijado, levantarse disciplinadamente al sonido de la campanilla, moverse en línea y realizar todos los movimientos con precisión. La evolución del trabajo, en el contexto de la revolución industrial avanzada, exigiría, más tarde, la enseñanza de competencias profesionales que por diferentes razones, (no nos vamos a ocupar ahora de analizarlas) seguía siendo masiva y corría a cargo de los presupuestos del Estado.

- En el momento actual nos encontramos ante una nueva coyuntura caracterizada por el valor mercantil del conocimiento. Vivimos una etapa de la historia, algunos la denominan una nueva era, en la que el conocimiento ha adquirido un extraordinario valor. Mientras la información abunda y se regala, el conocimiento de calidad es cada vez más escaso y más caro y para cuyo acceso vuelven de nuevo a establecerse barreras de tipo económico porque sólo pueden acceder los que pueden pagarlo. Nos estamos refiriendo al conocimiento superior del que se apropian los profesionales altamente cualificados, es aplicado en los sectores tecnológicos estelares y circula no en las universidades de masas sino en los centros elitistas de educación superior. Muchos anuncian que la valoración del conocimiento está ya lo suficientemente madura como para que pueda ser cotizado en bolsa y su expansión no tenga por qué depender de los recursos del Estado. Por otra parte, las nuevas tecnologías de la información y la comunicación favorecen la "comercialización" de la enseñanza y su puesta en circulación en la red del mercado de los servicios. Todo ello está haciendo posible que se esté pasando, en lo que se refiere al conocimiento de calidad, de la fase de la formación para todos a la de la formación para todos los que la puedan comprar o de la fase de las universidades de masa a la de las universidades de élite que son las que merecerán el verdadero título de centros superiores de enseñanza.

En este sentido se observa que los sistemas de educación superior, que hasta hace dos décadas giraban, según el triángulo de Clark, B. R. (1998), en torno al eje A/B (Estado-Corporaciones), han comenzado a acercarse al ángulo $\mathrm{C}$ que representa al mercado. 


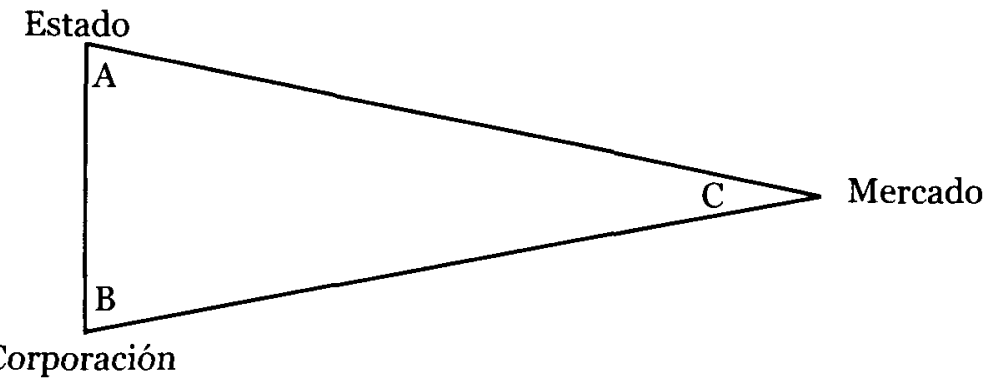

Frente al tradicional eje A/B en el que se desarrollaban las tradicionales tareas y actividades de los sistemas de enseñanza superior, están apareciendo y fortaleciéndose dos nuevos ejes orientados hacia el mercado. Por una parte el eje $\mathrm{B} / \mathrm{C}$ en el que las instituciones presentan entre sí una lucha competitiva por los mejores alumnos, los mejores profesores y el mayor número de recursos y el otro eje $\mathrm{A} / \mathrm{C}$ en el que los Estados desarrollan políticas educativas tendentes al mercado y a la formación en competencias prioritariamente productivas y rentables.

Un estudio comparado sobre la enseñanza superior publicado recientemente y coordinado por Brunner (2005) demuestra que, durante las dos últimas décadas, los sistemas de educación superior se han desplazado de tal forma hacia el polo del mercado que, en diversas partes del mundo, éstos dejan de funcionar como una parte del sector público sujeta a la conducción y administración del gobierno y se asemejan más a un mercado donde las instituciones compiten para ofrecer el servicio educacional.

\section{Ubicación de varios sistemas de educación superior en el continuo de coordinación gubernamental - de mercado*}
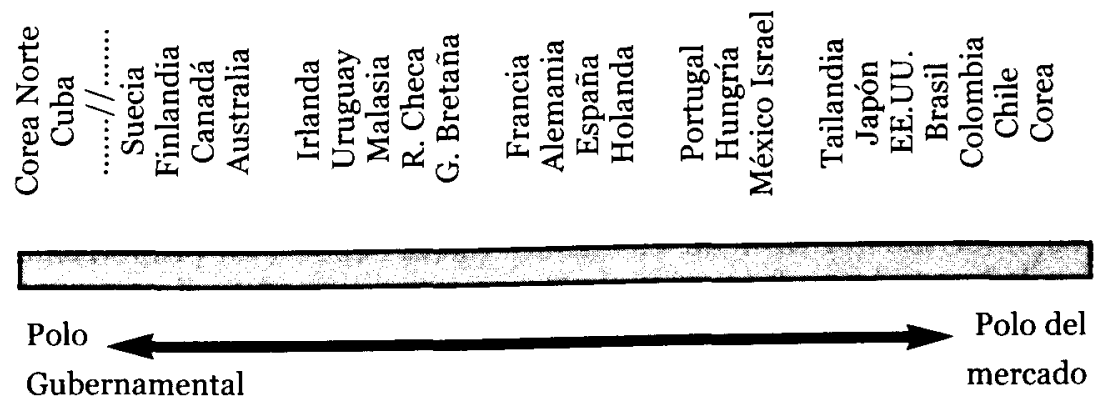

Fuente: Brunner y otros (2005). Guiar el Mercado. Informe sobre la Educación Superior en Chile. Universidad Adolfo Ibáñez. (Chile). 


\section{DE LA UNIVERSIDAD PÚBLICA A LA UNIVERSIDAD PRIVADA}

Siguiendo el análisis del Banco Mundial del año 2003 titulado Construir Sociedades de Conocimiento: Nuevos Desafios para la Educación Terciaria ${ }^{2}$ los medios económicos ejercen actualmente fuertes presiones sobre los servicios públicos para que éstos se adapten a las necesidades de la producción. En concreto, se pide al sistema de enseñanza que sea flexible y responda a los imperativos del mercado del trabajo. Todos los países pero especialmente los países en desarrollo están sometidos a grandes presiones para poder satisfacer la demanda de conocimiento superior que necesitan los sistemas productivos. En Brasil, por ejemplo, el incremento de matrícula en enseñanza superior ha sido más del 70\%. En China se establecieron más de 500 instituciones nuevas de enseñanza superior entre 1995 y 1999. Los gobiernos de países industrializados vienen invirtiendo en educación superior en torno al $15-20 \%$ del total que dedican a educación (entre un 4-6\% del PIB). Los países en vías de desarrollo dedican más del $20 \%$ de su presupuesto educativo al sector de la educación superior. Pero tanto unos como otros no pueden satisfacer la demanda con sólo los presupuestos fiscales, con mayor razón si las reformas liberales tienden a reducir la fiscalidad.

En un contexto de necesidades y demandas de conocimiento tan amplias, en el que los recursos públicos no son suficientes para abastecerlas, solamente caben dos salidas, o se aumenta la fiscalidad para que los Estados puedan afrontar dichos gastos o se encarga al mercado que satisfaga esta demanda. En una política mundial en donde domina la política del máximo al mercado no parece extraño que la educación y especialmente la educación superior quede integrada en el mercado internacional. Las negociaciones llevadas a cabo por la Organización Mundial del Comercio (OMC) para liberalizar el comercio de los servicios favorecen esta tendencia y es fruto al mismo tiempo de sus pretensiones. Desde 1995, El Acuerdo General sobre el Comercio de Servicios (AGCS) ha cambiado las perspectivas de la enseñanza superior como servicio. La formación, la capacitación superior y el conocimiento, en términos generales, constituyen desde entonces un mercado formal de orden internacional y determinados gobiernos ya han suscrito compromisos de liberalización en materia de servicios educativos o están invitando a las distintas empresas de formación a que se instalen en sus países. Los centros de formación superior son cada día más conscientes de que se están dirigiendo a consumidores que pueden elegir los mejores cursos existentes en el mercado.

Desde esta perspectiva se constata que la inversión de los gobiernos en educación superior, aunque aumente en algunos casos, tiende a ser proporcionalmente inferior a la inversión en formación de las empresas privadas. Si comparamos los gastos anuales de las empresas dedicados en el 2002 a la formación con los ocho países de la OCDE de los que se tienen datos disponibles, observamos que las empresas aumentaron la inversión en material intangible en el que está incluida la formación de 18 mil millones, en el año 1997, a 28 mil millones de dólares en el 2002 (Banco Mundial 2003). En cambio, de los ocho países de la OCDE de los cuales se tienen datos disponibles, en siete de ellos los gastos de origen privado para la educación 
superior terciaria han crecido en mayor proporción que los gastos públicos (salvo en Francia). En Canadá, Italia, Holanda y Suiza los presupuestos públicos han disminuido en términos reales.

Para hacernos una idea del crecimiento del sector privado en la enseñanza superior en todas las regiones del mundo podemos citar solamente algunos ejemplos:

- En Portugal las universidades privadas se han expandido en menos de una década al punto que hoy representan 30\% de las instituciones de educación terciaria, en las cuales está matriculado casi el 40\% del total de la población estudiantil.

- Un estudio reciente sobre educación terciaria en América Latina y el Caribe reveló que la acelerada expansión de la tasa de matrícula y la mayor diversificación institucional de la región no tuvieron su origen en esfuerzos del Estado sino que, más bien, han surgido como respuesta del sector privado a la creciente demanda social y a los cambios ocurridos en el mercado del trabajo (BID, 1999). Muchos países de la región han presenciado el impresionante auge de las instituciones de educación terciaria privadas en los últimos quince años pasados. En Brasil el 71\% de la matrícula en educación superior pertenece a centros privados. En la República Dominicana y El Salvador la tasa de matrícula estudiantil en instituciones terciarias del sector privado subió aproximadamente de 25\% en 1970 a cerca de 70\% en 1996 (García Guadilla, 1996).

- El cambio de inclinación en la balanza entre la oferta pública y la privada ha sido aún más pronunciado en los antiguos países socialistas de Europa Oriental y Asia Central, donde ha habido una evolución rápida del sistema centralizado hacia el liberalismo económico. En muchos países de la Europa Oriental en donde solamente hace diez años se desconocía la enseñanza superior privada, ahora está en pleno auge. En Polonia hay 195 centros de enseñanza superior con 377 mil estudiantes. El promedio de estudiantes en instituciones privadas en la República Checa, Hungría, Polonia y Rumania es del $22 \%$. En Filipinas y Corea el sector privado representa, respectivamente, el 80 y $75 \%$ del total de matrículas. Incluso en Bangladesh, donde no han comenzado a operar las Universidades privadas hasta 1992, éstas ya cuentan con $15 \%$ de la población estudiantil del país, y su crecimiento continúa.

- En los países del África subsahariana el número de instituciones educativas del sector privado creció aproximadamente de 30 en 1990 a más de 85 en 1999. En Costa de Marfil el número de estudiantes de los centros privados subió, entre los años 1991 a 1995, el $20 \%$ en la enseñanza primaria, el $33 \%$ en la secundaria, el $140 \%$ en la profesional y el $670 \%$ en la superior. 


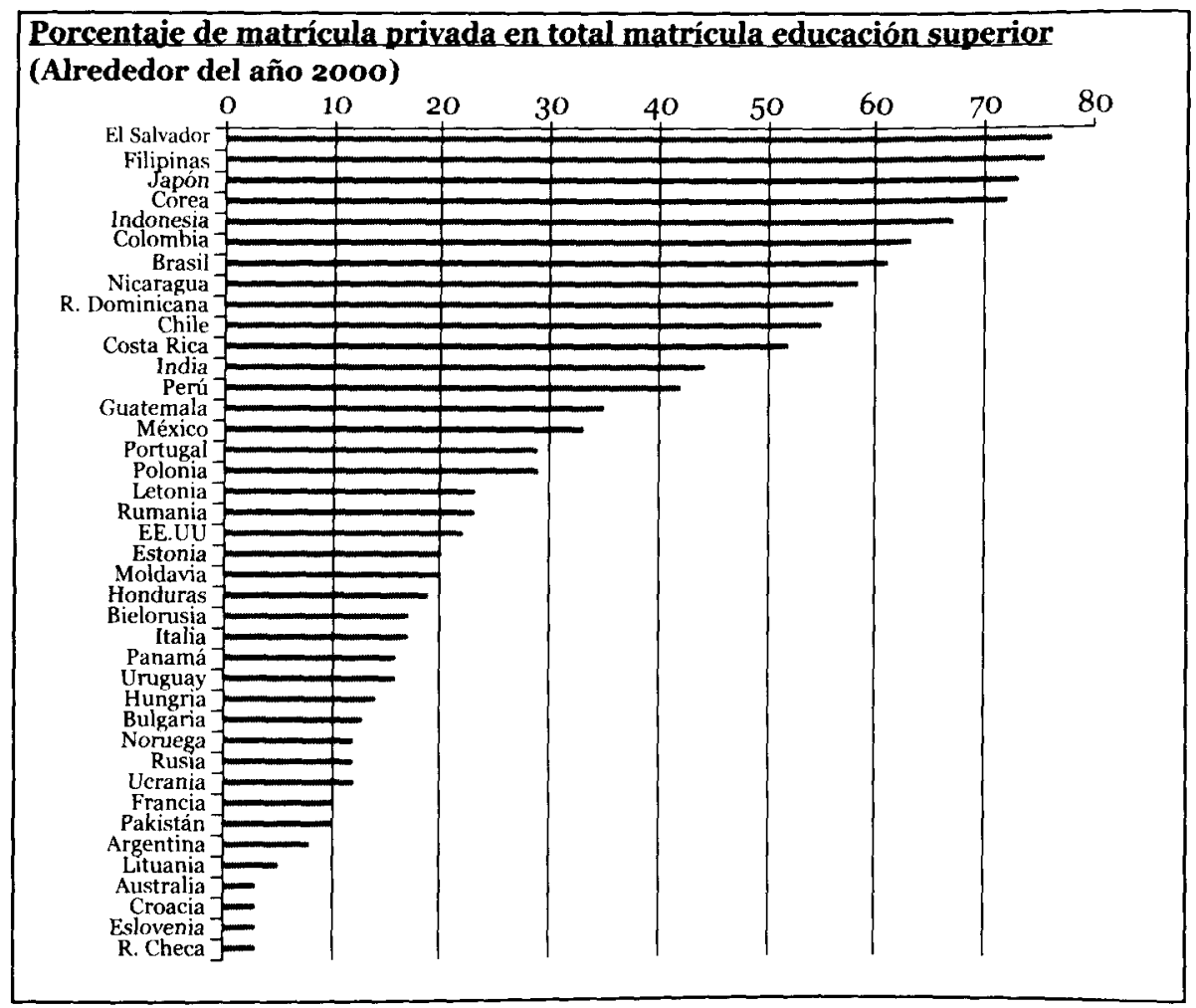

Fuente: Brunner y otros (2005). Guiar el Mercado. Informe sobre la Educación Superior en Chile. Universidad Adolfo Ibáñez. (Chile).

\section{LA ENSEÑNANA SUPERIOR EN LA RED DEL MERCADO}

En primer lugar hay que aclarar que no todas las instituciones privadas de enseñanza superior tienen fines lucrativos sino que existen también aquellas que se rigen por otros principios no lucrativos y que no se identifican totalmente con los fines del mercado. Nos estamos refiriendo a todo el ámbito de la denominada economía social en la que se pueden incluir fundaciones, cooperativas, ONGs, etc. Son empresas que no dependen directamente de la venta de sus productos sino de donaciones, impuestos voluntarios, etc. y que por lo tanto aunque vendan sus productos lo hacen con criterios sociales y no siempre al precio de su coste.

Dicho esto nos parece importante resaltar que muchas de los centros de enseñanza superior privados van en busca, en este momento más que en ningún otro, de una rentabilidad económica. Hasta tal punto esto es así que ya dos de las más grandes sociedades de Estados Unidos especializadas en formación superior, Apollo y Sylvan Learning, están cotizando en bolsa. 
Por otra parte, está apareciendo un tipo de universidades directamente ligadas a grandes empresas transnacionales. Hay que destacar la de Disney, Toyota y Motorola que funciona con un $4 \%$ de la nómina de la empresa, y administra 99 centros de aprendizaje en 21 países; $^{3}$ las de IBM y Dow Chemical que funcionan como universidades virtuales.

Están apareciendo, al lado del modelo tradicional de universidades, nuevas instituciones de educación superior como son, por ejemplo, universidades corporativas, universidades de franquicia, empresas editoriales educativas, empresas de comunicación masiva, proveedores internacionales etc. (Salmi, 2001). Este tipo de universidades, normalmente americanas, británicas o australianas, han comenzado a extenderse por Asia del Sur y Europa oriental. Actualmente existen en el mundo unas mil seiscientas universidades corporativas, algunas con autorización para dar títulos formales y otras no.

El mercado de la formación superior es, además, un mercado en auge. Según la banca americana Meryll-Lynch, para el año 2025 habrá el doble de estudiantes que existen en la actualidad, de 80 millones se pasará a 160.

La movilidad del estudiante buscando las mejores ofertas formativas es ya un hecho. A finales de los años noventa había más de un millón y medio de personas estudiando fuera de sus países en un mercado tasado por la OIT en 30 mil millones de dólares. Durante el año académico 2000-2001 hubo 547.867 estudiantes extranjeros en Estados Unidos y esto suponía una cifra aproximada del 3,8\% de la matrícula total en las universidades de Estados Unidos y una contribución a la economía americana de unos 11,04 mil millones de dólares. En 1998, se podía contar más de un millón trescientos mil estudiantes extranjeros en los países de la OCDE y un año más tarde eran más de un millón y medio. Es paradójico que en un momento en el que las tecnologías permiten estudiar sin salir de casa, la movilidad de estudiantes no ha sido nunca tan grande. Precisamente a este mercado es al que quiere acceder de una forma competitiva la Unión Europea y que forma parte del escenario que contextualiza el EEES.

Los países más poderosos compiten por obtener (comprar) personal altamente cualificado disponible en el mercado internacional. En el año 2000 Estados Unidos introdujo una enmienda a su ley de inmigración con el objetivo de poder expedir 600 mil visados a científicos. Algunas universidades europeas ya han planteado la dificultad de poder competir con las universidades americanas en los sueldos que éstas ofrecen a sus profesores. Cerca del $25 \%$ de los estudiantes de ciencia e ingeniería inscritos en programas universitarios de postgrado en Estados Unidos proviene de otros países, es decir, que entre 50 mil y 100 mil estudiantes extranjeros ingresan en el mercado norteamericano como capital humano avanzado y preparado en otros países.

Se estima, por ejemplo, que por lo menos $40 \%$ de los egresados de los prestigiosos institutos tecnológicos de la India buscan empleo en el exterior. Se estima 
que cerca de 30 mil africanos con título de doctorado viven fuera del continente africano y 130 mil de ellos se encuentran cursando estudios en el exterior. En el caso de Venezuela, $50 \%$ de los estudiantes egresados en el año académico 2000 de la Universidad Metropolitana, una de las más prestigiosas del país, fueron contratados por compañías multinacionales en otros países. En Bulgaria, la Unión Científica estima que $65 \%$ de los egresados universitarios (cerca de 300 mil personas) salieron del país en la última década.

Cuadro 1.2 Emigrantes con calificaciones de educación terciaria por regiones o países específicos, 1990

\begin{tabular}{lrcc}
\hline $\begin{array}{l}\text { País o región } \\
\text { de origen }\end{array}$ & $\begin{array}{l}\text { Número de } \\
\text { emigrantes que } \\
\text { residen en los EU }\end{array}$ & $\begin{array}{l}\text { Número de emigrantes } \\
\text { con educación terciaria } \\
\text { que viven en } U E\end{array}$ & $\begin{array}{l}\text { Proporción de emigrantes que } \\
\text { residen en EU con educación } \\
\text { terciaria sobre el total de } \\
\text { emigrantes (porcentaje) }\end{array}$ \\
\hline México & 2.700 .000 & 351.000 & 13 \\
Filipinas & 730.000 & & 50 \\
China & 400.000 & 200.000 & 50 \\
India & $300.000+$ & $225.000+$ & 75 \\
República de Corea & $300.000+$ & $159.000+$ & 53 \\
África subsahariana & 128.000 & 95.000 & 75 \\
Jamaica & & & 42 \\
Trinidad y Tobago & & & 46 \\
Sudamérica & & Aprox. 50 \\
\hline
\end{tabular}

Nota: Incluye únicamente inmigrantes a países de la OCDE; las cifras totales reales son probablemente más altas. Fuente: Carrington y Detragiache (1999).

\section{EL NUEVO PÚBLICO DE LAS INSTITUCIONES DE ENSEÑANZA SUPERIOR}

La procedencia de los estudiantes es cada vez menos lineal y nos encontramos con cantidades muy significativas de personas de 30 y 40 años que vuelven a estudiar desde el trabajo, hayan estudiado o no con anterioridad otra carrera. Esto quiere decir que la fuente de público que accede a la enseñanza superior no es solamente el publico juvenil que accede desde la enseñanza secundaria sino también un público adulto que accede desde las exigencias de su puesto de trabajo o desde sus responsabilidades e inquietudes socioculturales. Para el mercado de la enseñanza este público es más interesante en la medida en que la formación de los adultos es frecuentemente subvencionada en parte por las empresas y por lo tanto es una enseñanza muy rentable.

\section{ALGUNOS ELEMENTOS DE REFLEXIÓN SOBRE LA RELACIÓN ENTRE ECONOMLA Y EDUCACIÓN EN LA UNIÓN EUROPEA.}

La Mesa Redonda de los Industriales Europeos (ERT4) que es sin duda alguna la corporación más influyente en toda la política educativa de la Unión Europea, en los informes sobre la educación y la formación en Europa que este importante 
grupo de industriales comienza a hacer públicos a partir de 1989, incide reiteradamente en la necesidad de no dejar la formación en las manos exclusivas del mundo de la cultura académica ${ }^{5}$.

En su informe de 1994 sobre educación y formación, decía que se había acabado el tiempo en que los Estados reglamentaban la atribución de diplomas y que era necesario pasar a la "cartilla personal de competencias" destinada a convalidar las competencias adquiridas en el empleo y librada por organismos privados ${ }^{6}$. La ERT, especialmente interesada en convertir a las empresas en lugares de aprendizaje, plantea que estamos pasando de un modelo de enseñanza a un modelo de aprendizaje en el que los profesores ya no tendrán que transmitir conocimientos sino motivar, guiar y evaluar aprendizajes adquiridos por las vías más diversas. En 1995, en una reunión extraordinaria con el G7, (ERT, 1995) esta organización proclama que si la llave de la competitividad de Europa reside en sus niveles de conocimiento y competencia, la responsabilidad de la formación debe en definitiva ser asumida por la industria.

La influencia de la ERT en la Comisión Europea es tan grande que, como anota Nico Hirt, no se podría comprender en profundidad la política educativa de la Unión Europea sin conocer las relaciones con esta asociación de grandes industriales europeos. Efectivamente, tres años más tarde de la puesta en circulación de los primeros informes de la ERT, en 1992, el artículo 126 del tratado de Maastricht concede por primera vez competencias en materia de formación a la Comisión Europea. Se crea la Dirección General de Educación, Formación y Juventud (DGXXII) dirigida por Edith Cresson quien rápidamente encomienda al profesor Jean-Louis Reiffers la coordinación de un grupo de reflexión sobre la educación y la formación en Europa. Este grupo es el que elaborará el famoso libro blanco (1995) sobre Enseñar y aprender: hacia una sociedad cognitiva en donde se asumen todos los planteamientos más importantes de los industriales.

Las políticas de la Unión Europea sobre este tema se insinúan en este famoso libro blanco en donde se asumen todos los planteamientos más importantes de la cultura industrial y del sistema productivo. Es en este libro en el que se plantean con toda claridad las exigencias de la ERT en cuanto a dar prioridad a la formación en competencias, a que tanto la escuela como la empresa han de reconocerse como lugares de adquisición de saberes y que la necesidad de aprender a lo largo de toda la vida. Una de las más importantes recomendaciones de la ERT y que se hace prioritariamente desde la perspectiva de aprender a lo largo de toda la vida activa, es la de abrir nuevos modos de aprendizaje, de reconocimiento y de validación de competencias adquiridos en la experiencia laboral.

Desde la publicación de este libro blanco, en 1995, hasta, por poner un límite, la publicación del libro verde sobre el espíritu empresarial en Europa de 2003, la Unión Europea ha promovido insistentemente la necesidad de que la empresa se convierta en lugar de enseñanza y aprendizaje y que el aprendizaje a lo largo de la vida activa se favorezca con sistemas de validación de los aprendizajes adquiridos en la experiencia laboral. 
Por otra parte y desde la perspectiva de la comercialización de la enseñanza, la ERT, introduce nuevos planteamientos que conducen al trato comercial de la educación de la misma manera que, desde principios de los 80, Margaret Thatcher venía haciendo con otros servicios públicos como los teléfonos, los ferrocarriles, las compañías aéreas y servicios del agua.

Hemos de decir que la Unión Europea lleva varios años reflexionando sobre los Servicios de Interés General (S.I.G). Ello desembocó en la presentación, en mayo de 2003 , de un libro verde, en el que la Comisión Europea presentaba un balance de las reflexiones y de la atención prestada a los S.I.G. a escala comunitaria y nacional. Es muy útil seguir el debate producido a partir del Libro Verde sobre comercialización de Servicios de Interés General y sobre La Directiva del Parlamento Europeo y del Consejo relativa a los servicios en el mercado interior ${ }^{\beta}$, y el Libro Blanco sobre los servicios de interés general".

Un documento de interés, en el aspecto económico, es la comunicación de la Comisión de las Comunidades Europeas sobre Invertir eficazmente en educación y formación: un imperativo para Europa ${ }^{\prime \prime}$ en donde se promueve una mayor inversión privada para la formación.

Los países que más rápidamente descubren en la comercialización de la formación una fuente importante de ingresos son aquellos que más estudiantes extranjeros acogen en sus instituciones educativas ${ }^{11}$ como Estados Unidos, Australia, Japón y también algunos países europeos. El ministro francés de educación, Claude Allègre $^{12}$, presumía en 1998 de vender formación técnica al extranjero por valor de 2.000 millones de francos en tres años y estaba convencido de que la venta del conocimiento sería el gran mercado del siglo XXI. En un amplio debate sobre la relación entre la economía y la cultura, algunos autores han demostrado la potencialidad del mercado cultural en el que se incluye la enseñanza.

El periódico francés Le Monde $e^{44}$ publicó en el año 2001 un suplemento especial sobre las nuevas tecnologías y dedicó un gran espacio a la comercialización de la educación, en particular de la educación superior. Stéphane Medrad informaba en aquel número de la importancia que tenía el que Vivendi, la antigua compañia francesa del agua, hubiera invertido 25 millones de dólares para lanzar su portal de educación, una cantidad bastante inferior a la que el Banco Santander había ya invertido por aquellos años en Universia (no menos de 60 millones hasta 2001). En Estados Unidos las famosas EMOs (Education Management Organisations) gestionaban durante el curso 1999-2000 más de 285 escuelas y cotizaban en bolsa con el objetivo lógico y directo de obtener beneficios económicos. En Europa tenemos muestras parecidas como la de Educinvest ${ }^{15}$ que pertenece a Vivendi, y que ya en 1995 gestionaba unas 250 escuelas y movía anualmente más de 850 millones de francos (unos 130 millones de euros). En el reino Unido la sociedad Capital Strategies sacó a la bolsa, en 1996, UK Education y se enorgullece de tener altos rendimientos: una inversión de $1.000 £$, en el momento de lanzamiento (1996) valía en julio del año 2000 unas $3.405 £$ lo que representaba una rentabilidad de $240 \%$ (Niko Hirt: 2001 ). 


\section{LA CONTRAPARTIDA DEL MODELO DEL MERCADO}

Sobre la relación estrecha y a veces de dependencia entre economía y educación se han levantado voces críticas que merece la pena tener en cuenta si queremos tener una visión completa de las reflexiones que se hacen en torno a la enseñanza superior en Europa.

A título de ejemplo, podemos citar la disonancia interna que se está produciendo en el Partido Socialista Francés (PSF), de la que no se han hecho eco el resto de partidos socialistas europeos. El PSF ha divulgado la reflexión sobre la existencia de dos modelos de sociedad entre los que se debate Europa ${ }^{16}$. A su juicio, la disyuntiva está entre una Europa vieja que opta por la integración del modelo social europeo en el de la mundialización liberal, o el de una Europa de progreso y de justicia que integra la mundialización liberal en su modelo social. Otro ejemplo de reflexión crítica sobre la evolución de la educación en Europa se puede encontrar en diversas asociaciones y organizaciones internacionales ligadas a movimientos de tipo internacional como, por ejemplo, el Foro Social Europeo de octubre del 2004 que abordó temas como el de Otra educación es posible: oposición y resistencia al neoliberalismo.

En España existen movimientos críticos al pensamiento dominante de la UE sobre el EEES. Entre los universitarios, uno de los autores más conocidos es Carlos Taibo (2004), quien hace un análisis crítico a la Unión Europea realmente existente. Reflexiones críticas a añadir, en este caso directamente al EEES, las podemos encontrar en los profesores Teresa Gómez del Castillo y Antonio Aguilera, de la Universidad de Sevilla' ${ }^{17}$. La Revista Interuniversitaria de Formación del Profesorado se ha hecho eco de algunos análisis críticos en varios de sus números y especialmente dedicado al Congreso que sobre el Espacio Europeo de Educación Superior organizó en Segovia en el año 2005.

La contrapartida y crítica a la mercantilización de la enseñanza superior se centra fundamentalmente en tres aspectos:

\subsection{La equidad}

El principal problema del Consenso de Washington es que en él queda prácticamente excluido el tema de la equidad. Esta exclusión es grave, porque uno de los lugares en donde más se aplican las políticas de ajuste derivadas del consenso (e implementadas por el FMI) es en América Latina. Y éste es el continente más desigual del planeta: el PIB per cápita del $20 \%$ más rico es 18,7 veces el PIB per cápita del $20 \%$ más pobre, cuando en la OCDE esta diferencia entre los más ricos y más pobres es de 6,8 veces y la media mundial es de 7,1. (Informe PNUD del 1997).

Si se opta por disminuir la fiscalidad y los gastos estatales en determinados servicios y se anima a cubrirlos con los gastos de los hogares, la solidaridad con las 
familias y los individuos menos pudientes decrece hasta tal punto que muchos servicios básicos no pueden ser satisfechos por los necesitados. La pobreza aumenta y las barreras entre pobres y ricos se ensanchan. En cuanto a educación observamos que:

- Son notorias las desigualdades que el mercado del conocimiento no considera entre los países ricos y pobres en términos de inversión y capacidad científica y tecnológica. En 1996 se calculó que los países miembros de la OCDE representaban el 85\% de la inversión total en investigación y desarrollo; China, India, Brasil y los nuevos países industrializados de Asia Oriental el $11 \%$, y el resto del mundo sólo el $4 \%$.

- Existen crecientes disparidades en los recursos financieros entre las universidades públicas y privadas. De las veinte primeras universidades de Estados Unidos (según el estudio aparecido en US News and World Report correspondiente a 2001), sólo dos de ellas, la Universidad de California en Berkeley y la Universidad de Michigan, son públicas.

- En el mercado laboral global del profesorado universitario, los altos salarios pagados por universidades de un país pueden ejercer un impacto negativo en las instituciones de educación terciaria de otras partes del mundo y así contribuir a una injusta fuga de cerebros. Ni siquiera las mejores universidades de Europa están inmunes a esta amenaza, como lo demuestran las recientes quejas de las directivas universitarias de Gran Bretaña respecto a que ya no pueden ofrecer sueldos competitivos para atraer a especialistas eminentes a la profesión académica.

\subsection{Descuido de los problemas globales}

Los problemas del medio ambiente no se contemplan porque no se contabiliza como costo de producción el deterioro ecológico progresivo. En general, todos los problemas de largo alcance como el agua, la energía, el deterioro de la capa de ozono quedan postergados al actuar sistemáticamente bajo los efectos del corto plazo.

\subsection{Descuido de los problema radicales}

Se habla mucho de competencia y competitividad pero se dice menos de la necesaria tarea gubernamental de luchar para que se mantengan condiciones auténticas de competencia en los mercados.

El ex-presidente del Banco Mundial, James Wolfensohn, ha declarado recientemente que el mundo en el que vivimos está un poco enloquecido porque dedica 900.000 millones de dólares anuales en defensa y sólo 1.000 millones al desarrollo; porque se preocupa de las amenazas inmediatas y perdemos de vista las causas 
a largo plazo como la pobreza, frustración y falta de esperanza y porque no tiene 15 ó 20 líderes dispuestos a dedicar el $5 \%$ de su tiempo a tratar problemas globales (El País 8 de noviembre de 2004). También el Programa de Naciones Unidas para el Desarrollo (PNUD) cuyo administrador es Mark Malloch Brown, antiguo Director de Asuntos Externos del Banco Mundial (período 1994-1996) y Vicepresidente de Asuntos de las Naciones Unidas del Banco Mundial (período de 1996 a 1999) considera que la privatización no es la panacea del desarrollo y para que la expansión del comercio beneficie a los pobres hay que establecer reglas de juego más justas en el plano internacional y hay que introducir otros factores como la participación ciudadana o la libertad cultural.

\section{A MODO DE CONCLUSIÓN}

Sin tener nada en contra sobre la necesidad de aprender competencias, sobre todo en un mundo en el que los perfiles profesionales cambian tan rápidamente y en el que el trabajo sigue siendo la puerta principal para la integración social y para la supervivencia, pensamos que los estudios superiores no deben quedar bloqueados por uno de los ámbitos de nuestra sociedad, por muy importante que sea. Creemos que además de enseñar a trabajar nuestras universidades deben enseñar a pensar y por qué no a imaginar, a sentir y a soñar. Aunque el desarrollo de estas capacidades no las necesitemos para producir, seguro que las necesitaremos para vivir con dignidad. 


\section{NOTAS}

1.- BRUNNER, sociólogo de la Universidad de Oxford, es el director de la Escuela de Gobierno de la Universidad Adolfo Ibáñez, y director del Master en Gerencia y Políticas Públicas, ex director de la Facultad Latino Americana de Ciencias Sociales (FLACSO), actualmente trabaja como director del Programa de Educación de la Fundación Chile y es miembro del Consejo del Instituto Internacional de Planteamiento Educacional de la UNESCO. Acaba de coordinar (febrero de 2005) en la Universidad Adolfo lbánez, la publicación de un estudio títulado: Guiar el mercado. Informe sobre la educación superior en Chile.

2.- Seguimos el análisis del Banco Mundial del año 2003 titulado Construir Sociedades de Conocimiento: Nuevos Desafios para la Educación Terciaria. Se puede consultar en: http://www.bancomundial.org.mx/pdf/Educacion/LaEnsSup/4.pdf

3.- DENSFORD, L. (1999). Motorola University: The next 20 years. URL: http://www.traininguniversity.com/magazine/jan_feb99/feature1.html [Conferido em: 7 Fev. 2001].

4.- La Mesa Redonda Europea de Industriales (ERT) es un grupo en el que se encuentran organizados, desde 1983, los representantes de las principales industrias europeas como, por ejemplo, Peter Brabeck (Nestié), Paolo Fresco (Fiat), Leif Johansson (Volvo), Thomas Middelhoff (Bertelsmann), Peter Sutherland (BP) o Jürgen Weber (Lufthansa). A partir de 1989 forman una comisión específica de educación y formación que elabora informes educativos casi determinantes para la política educativa de la Unión Europea.

5.- Fn el informe Education et Compétence en Europe, de enero de 1989, la ERT publica un informe alarmante en el que se dice que "el desarrollo técnico e industrial de las empresas exige claramente una renovación acelerada de los sistemas de enseñanza y de sus programas". L'ERT lamenta que "la industria tenga tan poca influencia en los programas enseñados", que los docentes tengan "une comprensión tan insuficiente del entorno económico y que no comprendan las necesidades de la industria. Sin embargo, insiste la ERT, "competencia y educación son factores vitales de éxito". En conclusión, el poderoso grupo de presión patronal sugiere multiplicar los convenios entre escuelas y empresas" e invita a "tomar parte activa en el esfuerzo de la educación" y pide a los responsables de la política a "asociar a los industriales a todas aquellas debates que se refieran a la educación".

6.- Citado en LAVAL, C. (2004): La escuela no es una empresa. Edit. Paidos. Barcelona. Pág. 180.

7.- Bruselas 21/05/03 (COM 2003) 270 final. http://europa.eu.int/eur-lex/es/com/gpr/2003/com2003_o270eso1.pdf

8.- Bruselas 13/01/04 COM (2004) 2 final http://www.europa.eu.int/eur-lex/es/com/pdf/2004/com2004_0002eso1.pdf

9.- Bruselas 12/05/04 COM (2004) 374 final http://europa.eu.int/eur-lex/es/com/wpr/2004/com2004_o374es01.pdf

10.- http://europa.eu.int/eur-lex/es/com/cnc/2002/com2002_o779eso1.pdf

11.- Según el Compendio Mundial del la Educación 2005 Comparación de las Estadisticas de Educación en el Mundo. Instituto de Fstadística de la UNESCO, Montreal. http://www.uis.unesco.org/template/pdf/ged/2005/ged2005_sp.pdf Según este estudio los datos que ofrece para el curso 2002/2003 son los siguientes: Estados Unidos recibe 582.996 alumnos extranjeros de educación terciaria, Australia 179.619. Japón 74.892. Algunos países europeos también tienen un elevado número de alumnos extranjeros del nivel terciario: Alemania 240.619, Reino Unido 227.273, Francia 221.567. España recibe 53.639.

12.- Les Échos, 3 de febrero de 1998.

13.- Según Anthony Buzzelli, en "Economic Impact of the Arts and Cultura in Greater Washington" (2000), el sector cultura genera aproximadamente 20.000 empleos en la ciudad de Washington $\mathrm{DC}$

14.- Le Monde. Suplemento especial del 26 de septiembre de 2001.

15.- Les Echos. 21 de febrero de 1995.

16. Oui a l'Europe federale et sociale, non a la constitution Giscard. http://www.parti-socialiste.fr/list theme.php?theme=MTYo

17.- Una de sus publicaciones: Perspectivas de la universidad española én el espacio europeo de educación superior, la podemos encontrar en internet.

http://www.ull.es/publicaciones/latina/20041257aguilera.htm 


\section{REFERENCIAS BIBLIOGRÁFICAS}

Banco Mundial (2003). Aprendizaje Permanente en la economía global del conocimiento. Desafios para los países en desarrollo. Alfaomega Grupo Editor.

Clark, R. B., (1998). Creating Entrepreneurial Universities. Organizacional Pathways of Transformation. Oxford; Pergamon - IAU Press.

García Guadilla, C. (1996). Conocimiento, Educación Superior y sociedad en América Latina. Caracas: Ed. Nueva SociedadCENDES.

Hirt, N. (2001). Los tres ejes de la mercantilización escolar. Disponible en: http://www.aep.pangea.org

Hirt, N. (2003). Los nuevos amos de la escuela. El negocio de la enseñanza. Madrid; Minor Network S.L. Editorial Digital.

Laval, C. (2004). La escuela no es una empresa. El ataque neoliberal a la enseñanza pública. Barcelona; Paidos.

Navarro, V. (Coord.) (2004). El Estado de Bienestar en España. Barcelona; Universidad Pompeiu Fabra.
Navarro, V. (2006). El subdesarrollo social de España. Causas y consecuencias. Barcelona: Anagrama.

OCDE, (2001). Análisis de las políticas de la educación. Disponible en:

http://www.1.oecd.org/publications/e_bbook /9601032e.pdf

Pardo, J. L. (2005). La dudosa modernización de la educación superior. Diario El Pais (21/03/05).

Salmi, J. (2001). La educación superior en un punto decisivo. Ponencia presentada al Congreso de "Educación Superior, desafío global y respuesta nacional", Universidad de Los Andes, Santa Fé de Bogotá, junio 21 y 22.

Taibo, C. (2004). No es lo que nos cuentan. Una crítica a la Unión Europea realmente existente. Madrid: Ediciones B.S.A. 


\section{PALABRAS CLAVE}

Enseñanza superior europea. Servicio público. Enseñanza y Mercado.

\section{KEY WORDS}

European higher education. Public service. Free market and teaching.

\section{PERFIL ACADEMICO DEL AUTOR}

Florentino Sanz Fernández, Profesor Titular de Departamento de Historia de la Educación y Educación Comparada. Sus líneas de investigación están vinculadas a la formación de personas adultas y educación popular.

Dirección del autor: Dpto. de Historia de la Educación y Educación Comparada. Facultad de Educación. UNED.

Edificio de Humanidades

Paseo Senda del Rey, 7

28040 Madrid

E-mail: fsanz@edu.uned.es

Fecha recepción del artículo: 03. abril. 2006

Fecha aceptación del artículo: 19. junio. 2006 